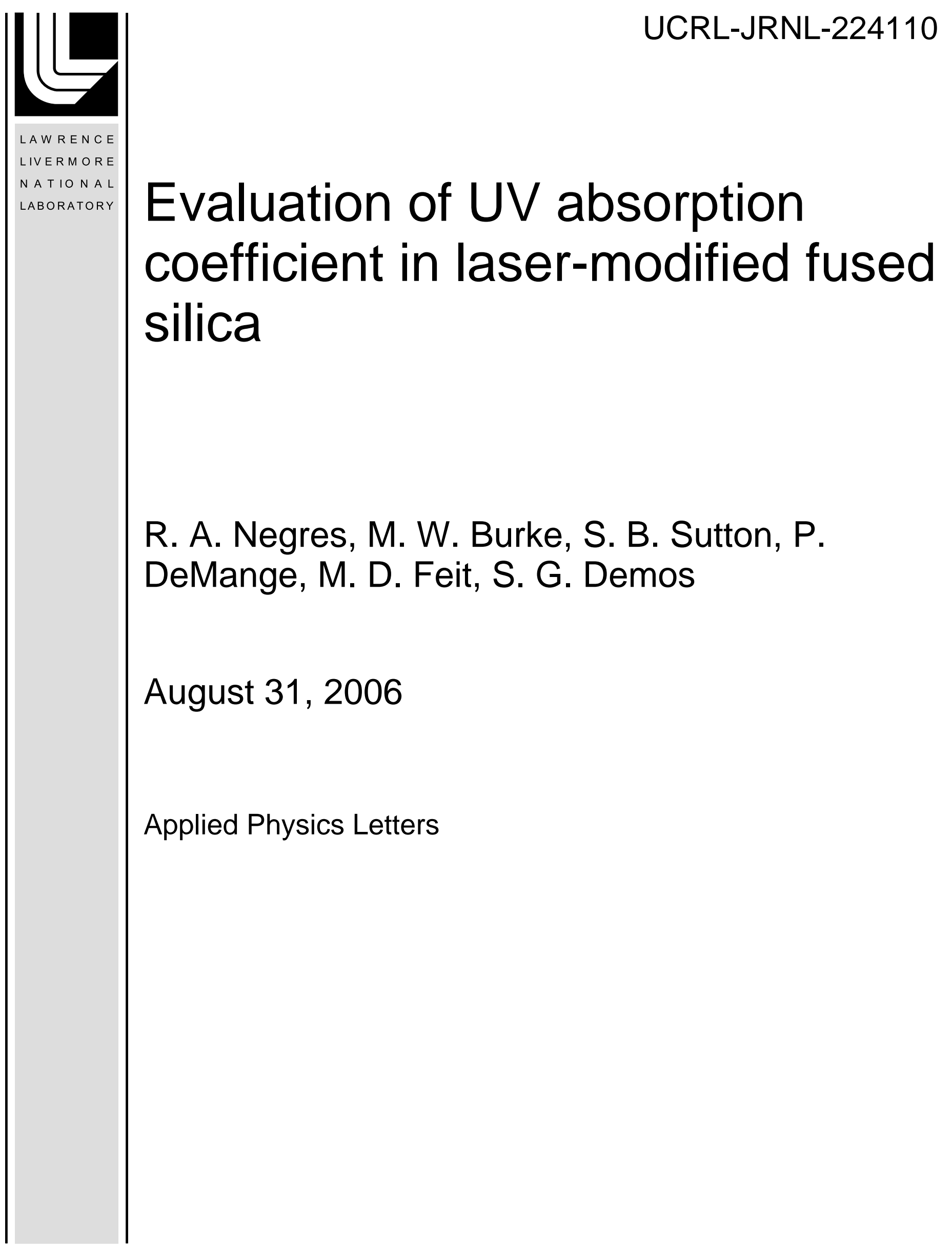


This document was prepared as an account of work sponsored by an agency of the United States Government. Neither the United States Government nor the University of California nor any of their employees, makes any warranty, express or implied, or assumes any legal liability or responsibility for the accuracy, completeness, or usefulness of any information, apparatus, product, or process disclosed, or represents that its use would not infringe privately owned rights. Reference herein to any specific commercial product, process, or service by trade name, trademark, manufacturer, or otherwise, does not necessarily constitute or imply its endorsement, recommendation, or favoring by the United States Government or the University of California. The views and opinions of authors expressed herein do not necessarily state or reflect those of the United States Government or the University of California, and shall not be used for advertising or product endorsement purposes. 


\title{
Evaluation of UV absorption coefficient in laser-modified fused silica
}

\author{
R. A. Negres, ${ }^{*}$ M. W. Burke, S. B. Sutton, P. DeMange, M. D. Feit, and S. G. Demos \\ Lawrence Livermore National Laboratory, \\ 7000 East Avenue, Livermore, California 94550-0808
}

(Received

\begin{abstract}
Laser-induced damage in transparent dielectrics leads to the formation of laser-modified material as a result of exposure to extreme localized temperatures and pressures. In this work, we used an infrared thermal imaging system in combination with a fluorescence microscope to map the dynamics of the local surface temperature and fluorescence intensity under cw, UV excitation of laser-modified fused silica within a damage site. Based on a thermal diffusion model, we estimate the energy deposited via linear absorption mechanisms and derive the absorption coefficient of the modified material. In addition, irreversible changes in the absorption following extended laser exposure were observed.
\end{abstract}

PACS numbers: 42.70.-a, 61.80.Ba, 78.20.Ci 
Laser-induced breakdown in the bulk of transparent dielectric materials creates extreme conditions associated with the generation of high temperatures and pressures. ${ }^{1,2}$ In large bandgap materials, such as fused silica $\left(\mathrm{SiO}_{2}\right)$, laser intensities in excess of $10^{12} \mathrm{~W} / \mathrm{cm}^{2}$ are required to achieve intrinsic breakdown. ${ }^{3}$ However, defects in the material can initiate localized breakdown at more than two orders of magnitude lower intensities. This latter process can lead to laser-induced damage (LID) and represents a major challenge in the manufacturing of optical materials for high power laser systems. The material directly exposed to these extreme conditions is mechanically and structurally modified. The modifications are in part manifested in the Raman scattering and photoluminescence spectra and are consistent with material densification and the formation of various defects species. ${ }^{4-6}$ Moreover, as recent studies in $\mathrm{SiO}_{2}$ have indicated, such changes are similar for laser-induced breakdown created by either femtosecond or nanosecond pulses. ${ }^{7}$ It has been even proposed that the photoluminescence of the modified material can be used for three-dimensional optical memory recording and reading. ${ }^{8}$ It is also possible that absorption by this modified material can affect the performance of optoelectronic devices manufactured via laser micro-machining. 9,10 In the case of optical components for high-power lasers, the presence of localized absorption at LID sites can lead to additional damage under subsequent laser irradiation (commonly referred to as damage growth). ${ }^{11}$ This is of critical importance in large-aperture laser systems where localized damage (on the order of a few tens of microns or less) will not inhibit the operation of the laser but subsequent growth of these sites can significantly increase the cost of operation. The layer thickness of modified material within LID sites in $\mathrm{SiO}_{2}$ formed under nanosecond laser pulse irradiation has been estimated to $\sim 10 \mu \mathrm{m} .{ }^{12,13}$ Photothermal microscopy has indicated the presence of absorption at such sites ${ }^{14}$ but, to the best of our knowledge, there is no precise estimation of the linear absorption coefficient of this modified material.

The objective of this work is to quantify the energy deposited within laser-modified fused silica via linear absorption mechanisms. Our approach involves the use of an infrared thermal imaging system to map, with adequate spatial and temporal resolution, the dynamics of the local surface temperature of the sample under cw, UV excitation. An increase in temperature may arise from energy dissipation through the non-radiative relaxation pathways by the absorbing defects created within the modified material during a damage event. A fluorescence microscope is concurrently used to image the same sites in order to also monitor 
the radiative relaxation pathways.

Typical LID sites at the exit surface of a fused silica (Suprasil II) substrate were prepared (in air, at room temperature) using 7-ns (FWHM), 355-nm laser pulses from a Q-switched Nd:YAG laser system as follows: each site was initiated with one pulse at $20-30 \mathrm{~J} / \mathrm{cm}^{2}$ and subsequently grown to $\sim 500-800 \mu \mathrm{m}$ in diameter using ten pulses at $10 \mathrm{~J} / \mathrm{cm}^{2}$. A multi-line (351-363 nm), cw Argon laser beam was centered and focused within a single damage site (Gaussian beam profile with HW1/eM of $110 \mu \mathrm{m})$. A mid-IR $(3-5 \mu \mathrm{m})$ imaging camera ${ }^{15}$ equipped with $\times 1$ magnification optics was positioned behind the sample to directly image its back surface to spatially resolve changes in the surface temperature prior to, during and after laser irradiation. The temperature calibration of the IR camera was performed for each acquisition configuration (frame rate and size, integration time) using a black body radiation source to derive the relative emissivity curve for our sample. In addition, we used a thermo-couple device to directly measure the temperature of the IR-imaged sample while it was heated from 20 to $60{ }^{\circ} \mathrm{C}$. The two calibration methods were in good agreement over this temperature range. In addition to monitoring the temperature, we also captured the fluorescence signal originating at LID sites during UV excitation using a fluorescence microscope placed in front of the sample, opposite to the IR camera. A schematic of the experimental setup is shown in Fig. 1 (top) along with typical fluorescence [Fig. 1(a)] and thermal images of one LID site [Figs. 1(b) and 1(c), prior to and during laser irradiation, respectively]. It is worth noting that the damage site is visible in Fig. 1(a) even without laser illumination due to perhaps its larger surface area compared to that of a pristine $\mathrm{SiO}_{2}$ surface.

Real time thermal imaging confirmed that, following the onset of laser irradiation, a temperature gradient is rapidly established throughout the substrate due to diffusion of energy deposited within the modified material. Experiments were performed in two different irradiation configurations, either short or extended exposure to UV laser beam with constant power.

In the short laser exposure regime, the sample was irradiated for few seconds. In order to adequately resolve the temperature rise and fall when the laser is turned $\mathrm{ON}$ and $\mathrm{OFF}$, respectively, maximum spatial resolution thermal maps were sequentially acquired at a high rate of 100 frames/sec with 0.5 msec integration time over 12 seconds including prior to, during and after the laser exposure (i.e., a total 1200-frame movie). The local surface 
temperature at various locations surrounding the damage site can then be extracted from individual frames and plotted as a function of time. As an example, Fig. 2 illustrates the measured temperature change at the center, $0.5 \mathrm{~mm}$ and $1.75 \mathrm{~mm}$ away from the LID site (locations 1, 2 and $\mathbf{3}$ in the inset thermal image, respectively) upon constant, 2.6-W irradiation. The temperature values represent averages over 4 pixels $\left(2 \times 2\right.$, or $\left.100 \mu \mathrm{m}^{2}\right)$ at these locations.

In order to derive the fraction of the incident energy that is deposited within the LID site following $\mathrm{cw}$ laser excitation and estimate the linear absorption coefficient of the modified material, we use a thermal diffusion model to fit the kinetics of the local surface temperature. A 2-D, radially axi-symmetric numerical model was constructed based on implicit time integration with a variable time step, a graded mesh in both $r$ - and $z$-directions and a Gaussian heat source profile (HW1/eM of $200 \mu \mathrm{m}$ to account for scattering-induced broadening of the illumination beam profile) with exponential absorption. The solid lines in Fig. 2 represent the best fits to the data, assuming a $500 \mu \mathrm{m}$ diameter LID site and a uniform absorption coefficient of the modified material within the site of $96 \pm 3 \mathrm{~m}^{-1}$ over a depth of $5 \mu \mathrm{m}$ (i.e., $0.048 \%$ absorption). ${ }^{12}$ The same experiment and numerical fitting of the data were repeated at five different LID sites and revealed values of the absorption coefficient of laser-modified material in the range $50-150 \mathrm{~m}^{-1}$. The spread in the values of the estimated absorption can be understood in terms of site-to-site variation in damage morphology as well as uncertainty in the depth of the absorption layer of modified material.

The numerical model described above can be used to predict the increase in temperature at the LID site under nanosecond, pulsed excitation assuming linear absorption at ICF laser fluences. This issue relates to the amount of damage growth upon subsequent laser exposure. Namely, if we consider an average UV linear absorption coefficient of the modified material of $100 \mathrm{~m}^{-1}$ and a $10-\mathrm{J} / \mathrm{cm}^{2}, 10$-ns incident laser pulse at $355 \mathrm{~nm}$ (Gaussian spatial beam profile with FHW1/eM of $500 \mu \mathrm{m}$ ), the model predicts an upper bound on the induced temperature rise at the LID site of $\sim 3{ }^{\circ} \mathrm{C}$. This value is significantly lower than what is required (on the order of $\sim 1000{ }^{\circ} \mathrm{C}$ or higher) to attribute the damage growth process entirely to uniform linear absorption in a modified layer of $\sim 10 \mu \mathrm{m}$ (leading to excessive heating of the material and subsequent dramatic change in its absorption characteristics). It is natural to think that absorption in the damaged material may be more localized and/or that nonlinear absorption mechanisms may be involved. 
The second irradiation regime involved the 'saturation' of the modified material within a site under constant UV laser illumination for an extended period of time (15 mins). During this time, a more significant heating of the entire sample was achieved. Simultaneously, the fluorescence microscope revealed a decrease in the fluorescence intensity originating at the damage site versus time. At the end of the 'saturation' period, the laser was turned off and the 'relaxation' of the sample's surface temperature back to room temperature was observed. In this irradiation regime, we have acquired thermal and fluorescence maps of previously non-irradiated LID sites approximately once every minute up to one hour (including both 'saturation' and 'relaxation' periods) with 0.5 msec integration time. During the 'relaxation' period, the laser was briefly turned on to acquire fluorescence images.

Figure 3 illustrates the average temperature and fluorescence intensity extracted from the corresponding maps of a fresh LID site both during and after a 15-min UV irradiation (at 2.7 W). For better signal-to-noise ratio, the signals were spatially integrated over the extent of the damage site hence the apparent slower dynamics as compared to that illustrated in Fig. 2 for short laser exposure regime. We note from Fig. 3 that i) during 'saturation', the average surface temperature increases by $\sim 1.5^{\circ} \mathrm{C}$ while the fluorescence signal rapidly decreases to $\sim 20 \%$ of its value prior to irradiation, and ii) after the laser is turned OFF, within 15 mins, the surface temperature at the site relaxes back to room temperature but the fluorescence signal remains low. The later is true even after one day, suggesting that extended UV laser exposure induces irreversible photochemical reactions that in turn lead to significantly lower absorption within the modified material. This assumption was verified by performing short laser exposure experiments (few seconds) at previously non-irradiated damage sites to measure the transient surface temperature both prior to and after extended UV irradiation. Indeed, these tests confirmed that the maximum temperature rise at LID sites following the 'saturation' and 'relaxation' periods is also reduced to $\sim 20 \%$ of its value prior to irradiation, consistent with a reduced absorption within the modified material (results are not shown here). This effect may be related to previous studies where it has been suggested that thermal annealing of fused silica leads to passivation of the defects associated with photoluminescence at optically produced damage sites. ${ }^{16,17}$ Similarly, a reduction in the nonlinear absorption at damage sites has been reported following prolonged exposure to pulsed laser radiation. ${ }^{18}$ It is not clear whether or not the same absorbing defect species and/or absorption mechanisms are involved in these processes. 
We thank J. D. Bude for useful discussions. This work was performed under the auspices of the U.S. Department of Energy by University of California, Lawrence Livermore National Laboratory under contract no. W-7405-Eng-48.

* Electronic address: negres2@llnl.gov. 


\section{References}

1 C. W. Carr, H. B. Radousky, A. M. Rubenchik, M. D. Feit, and S. Demos, Phys. Rev. Lett. 92, 087401 (2004).

2 S. Juodkazis, K. Nishimura, S. Tanaka, H. Misawa, E. G. Gamaly, B. Luther-Davies, L. Hallo, P. Nicolai, , and V. T. Tikhonchuk, Phys. Rev. Lett. 96, 166101 (2006).

3 B. C. Stuart, M. D. Feit, S. Herman, A. M. Rubenchik, B. W. Shore, and M. D. Perry, Phys. Rev. B 53, 1749 (1996).

4 M. Watanabe, S. Juodkazis, H.-B. Sun, S. Matsuo, and H. Misawa, Phys. Rev. B 60, 9959 (1999).

5 M. R. Kozlowski, C. L. Battersby, and S. G. Demos, Proc. SPIE 3902, 138 (1999).

6 S. G. Demos, M. Staggs, K. Minoshima, and J. Fujimoto, Opt. Exp. 10, 1444 (2002).

7 S. O. Kucheyev and S. G. Demos, Appl. Phys. Lett. 82, 3230 (2003).

8 Y. Li, W. Watanabe, K. Itoh, and X. Sun, Appl. Phys. Lett. 81, 1952 (2002).

9 Y. Cheng, K. Sugioka, and K. Midorikawa, Opt. Lett. 29, 2007 (2004).

10 T. Kurobori, T. Yamakage, Y. Hirose, K. Kawamura, M. Hirano, and H. Hosono, Jpn. J. Appl. Phys. 44, 910 (2005).

11 S. G. Demos, M. Staggs, and M. R. Kozlowski, Appl. Opt. 41, 3628 (2002).

12 M. A. Stevens-Kalceff, and J. Wong, J. Appl. Phys. 97, 113519 (2005).

13 J. Wong, J. L. Ferriera, E. F. Lindsey, D. L. Haupt, I. D. Hutcheon, and J. H. Kinney, J. Non-cryst. Solids 352, 255 (2006).

14 F. Bonneau, P. Combis, J. L. Rullier, M. Commandré, A. During, J. Y. Natoli, M. J. Pellin, M. R. Savina, E. Cottancin, and M. Pellarin, Appl. Phys. Lett. 83, 3855 (2003).

15 Model Phoenix 9803 (Indigo Systems), 640 x 512 cooled InSb sensor, $25 \mu \mathrm{m} /$ pixel, 14-bit dynamic range, full frame acquisition rates of up to 107 frames per second.

16 M. Watanabe, S. Juodkazis, H.-B. Sun, S. Matsuo, H. Misawa, M. Miwa, and R. Kaneko, Appl. Phys. Lett. 74, 3957 (1999).

17 S. Juodkazis, M. Watanabe, H.-B. Sun, S. Matsuo, J. Nishii, and H. Misawa, Appl. Surf. Sci. 154, $696(2000)$.

18 A. D. Walser, S. G. Demos, M. Etienne, and R. Dorsinville, Opt. Comm. 240, 417 (2004). 


\section{List of Figure Captions}

FIG. 1: Thermal and fluorescence imaging experimental setup. Surface damage sites on a $\mathrm{SiO}_{2}$ substrate were imaged prior to, during and after $c w$ UV laser irradiation. A fluorescence image of a representative LID site is illustrated in (a) while thermal maps of the same site are shown in (b) and (c) (with laser OFF and $\mathrm{ON}$, respectively).

FIG. 2: Change in surface temperature prior to, during and after a short UV laser irradiation (at $2.6 \mathrm{~W}$ ) of the modified material within a typical LID site at three different locations (labelled $\mathbf{1}$, $\mathbf{2}$ and $\mathbf{3}$ in the inset thermal image with laser ON). The solid lines represent numerical fits to the data based on a thermal diffusion model with the parameters described in the text.

FIG. 3: Average temperature and integrated fluorescence at a typical LID site during and after an extended, 15-min UV laser irradiation at $2.7 \mathrm{~W}$ (SATURATION and RELAXATION, respectively). 


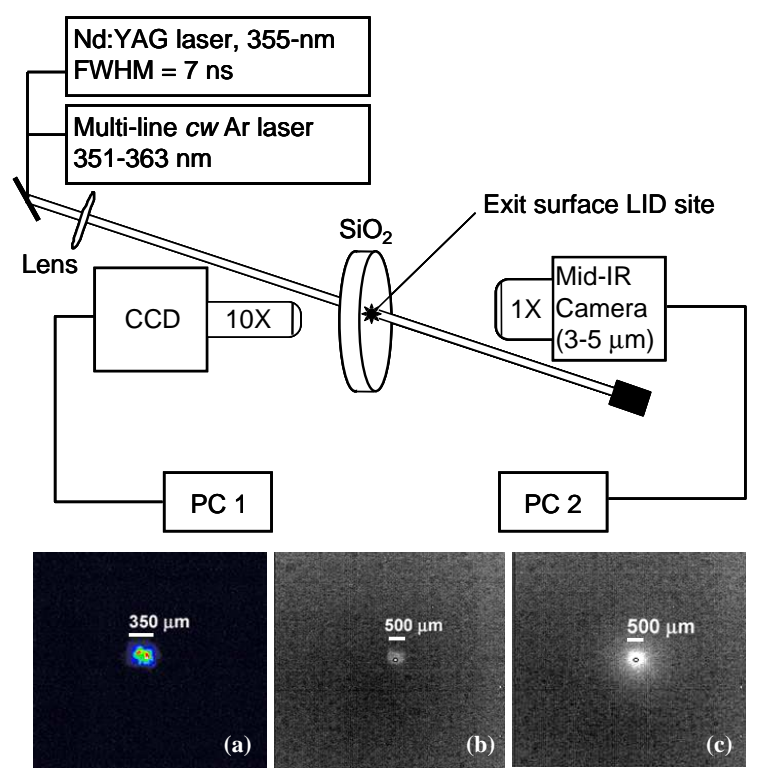

Figure 1. R. A. Negres et al. 


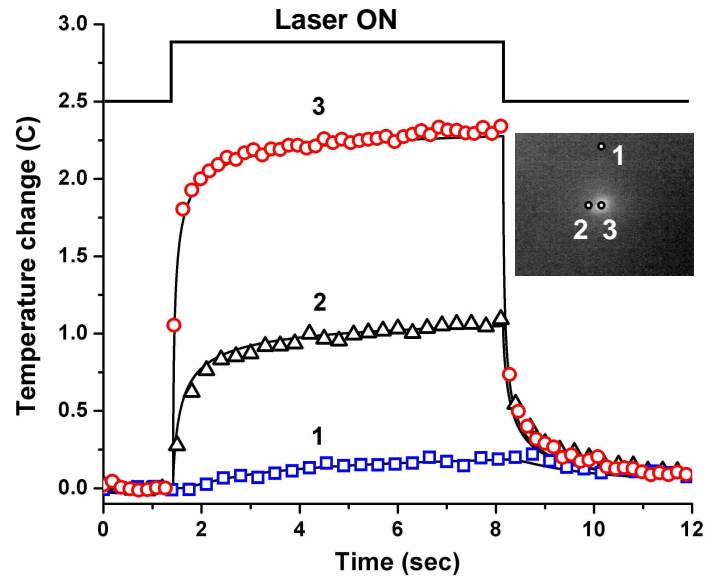

Figure 2. R. A. Negres et al. 


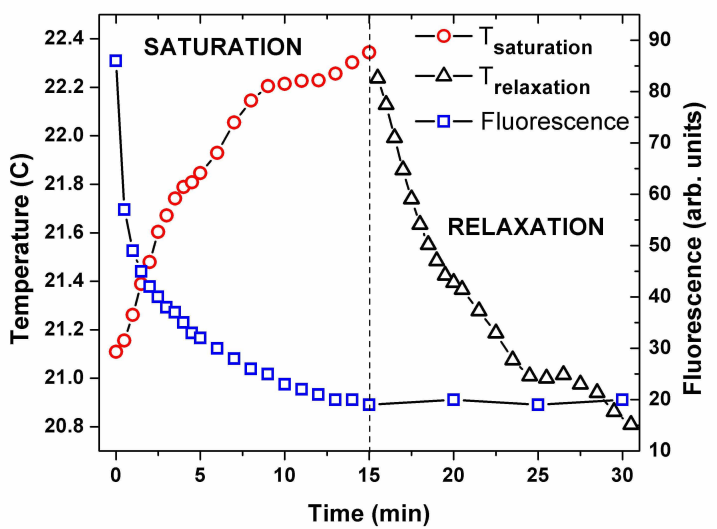

Figure 3. R. A. Negres et al. 